\title{
A PVDF-BASED FLEXIBLE AND SHAPEABLE ACOUSTIC POWER SOURCE FOR IMPLANTABLE BIOMEDICAL DEVICES
}

\author{
J. Zhou , R. Rahimi, A. Kim, M. Ochoa and B. Ziaie \\ Purdue University, West Lafayette, Indiana, USA
}

\begin{abstract}
Piezoelectric materials have been increasingly investigated over the past decade for energy harvesting and remote acoustic/ultrasonic/mechanical powering. In particular, wireless powering of implantable devices with sound and/or mechanical waves provides certain advantages compared to the traditional inductive method, mainly increased range, omni-directionality, and increased efficiency at $\mathrm{mm}$ and sub-mm scales. In this paper, we present a low-cost method for fabricating polyvinylidene fluoride (PVDF) power sources in form factors that are easily insertable with a biopsy needle and can form a desired shape once inside the body via a shape-memory alloy (SMA) wire. In-vitro experiments using receivers with $1 \times 2 \mathrm{~cm}^{2}$ in dimensions driven by an acoustic transmitter with an output intensity of $92 \mathrm{~dB}$ at $250 \mathrm{~Hz}$ results in an electrical output power of $9.5 \mathrm{nW}$.
\end{abstract}

\section{INTRODUCTION}

The recent proliferation of low-power electronics has been one of the main driving forces behind the renewed interest in energy scavenging as a mode of powering for implantable medical devices [1]. Batteries and inductive powering have been the mainstay of power generation for decades [2-4]. Due to their superior reliability, batteries have been the power source of choice for most low-power long-term implantable systems (e.g., cardiac pacemakers). However, batteries are still large, have non-desirable form factors, and surgical intervention is necessary when depleted and needs to be replaced. As an alternative approach, inductive powering has been the method of choice in satiations where power is not needed continuously and where surgical replacement is not easily feasible. Nevertheless, inductive powering suffers from its own shortcomings such as low misalignment tolerance and short transmission range.

Acoustic/ultrasonic powering can resolve some of the abovementioned shortcomings associated with batteries and inductive methods [5-6]. These include larger tissue penetration depth, omnidirectionality, and better efficiency at smaller receiver dimensions (mm and sub-mm) [7]. Vibration and acoustic energy, as one of the most available ambient energy sources, is also a promising format for energy harvesting. Lead zirconate titanate (PZT), due to its superior electromechanical coupling coefficient $(k)$, is the most widely used piezoelectric material in ultrasonic/acoustic systems; yet, concerns regarding possible leaching of lead have impeded its adoption for implantable applications. Polyvinylidene fluoride (PVDF) with its biocompatibility, flexibility, better acoustic matching to the soft tissue, and wide bandwidth, have become an attractive alternative candidate (at the expense of lower $k$ ). Nevertheless, issues regarding its incompatibility with many standard processing methods, 3D shaping, and difficulty of insertion into the tissue still pose challenges.

In this work, we present a low-cost benchtop method for the fabrication of an implantable PVDF power source in a size and form factor that can be easily implanted through a simple biopsy needle. In addition, by using a shape memory alloy wire, the PVDF can be automatically reshaped into a predefined geometry, once inserted.

\section{OPERATION PRINCIPLE}

Figure 1 shows the schematic of the PVDF power source with a SMA wire backbone in the bore of a biopsy needle. The device consists of a narrow strip of PVDF with a SMA wire (here thereafter referred to as a skewer) pierced through several micro-machined slits. The PVDF skewer can be implanted into soft tissue or body cavities (including vascular system) using a biopsy needle (Figure 1, left-side). Once the rolled PVDF is properly implanted in-situ, the unfolding and reshaping process start immediately after the removal of biopsy needle when the surrounding temperature reaches $37^{\circ} \mathrm{C}$. When in place, the PVDF strip is driven into vibration by an outside acoustic source. The resonant frequency of the PDVF receiver depends on its shape, dimensions, and material properties. When the vibration is considered as bending mode, due to the Young's Modulus of PVDF is $8.3 \mathrm{GPa}$, the first resonant frequency for the mm-scale dimensions is expected to lie within the range of audible sound.

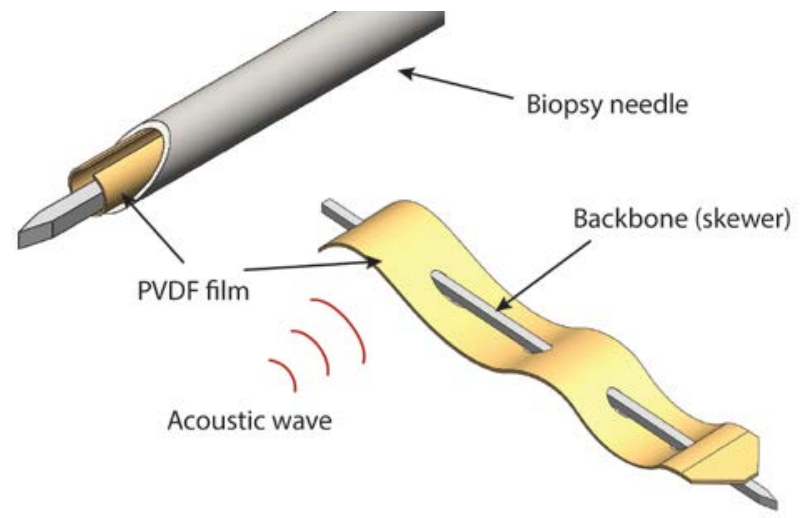

Figure 1: Schematic of a PVDF strip with an insertion-wire/skewer; the device inside a biopsy needle.

\section{FABRICATION}

The fabrication process of the PVDF power source is shown in Figure 2a. It starts with patterning a $110 \mu \mathrm{m}$-thick PVDF sheet (gold electrodes deposited, Precision Acoustics Ltd, UK) into a narrow strip with multiple transverse slits using a tabletop CAMEO ${ }^{\circledR} \mathrm{craft}$ cutter plotter (Silhouette America Inc, Utah, USA). This technique allows for simple and inexpensive machining of PVDF, which is notoriously difficult to process using traditional micromachining technologies. At this step, a small mass can be attached to the PVDF strip to increase the displacement. Then, a shape memory alloy wire (skewer) (Kellogg's Research Labs, USA) is stitched through the slits. SMA wire can be fixed to the PVDF using silver paint (Ted Pella Inc., USA) at room temperature to protect the piezoelectric property of PVDF. Finally, the entire device is coated with $5 \mu \mathrm{m}$ parylene-C for electrical passivation. Figure $2 b$ shows a fabricated prototype with both a $1.5 \mathrm{~mm}$ diameter biodegradable 3D-printed polylactic acid (PLA) (NinjaFlex, NinjaTek, USA) and SMA skewer inside a biopsy needle (inner diameter of $2.8 \mathrm{~mm}$ ). Figure $2 \mathrm{c}$ shows the PVDF with a SMA skewer before and after exposure to $37^{\circ} \mathrm{C}$, illustrating the possibility of shaping the device in-situ, once inserted.

To increase the output power of proposed design, multiple PVDF films can be stacked with the poling directions of each layer aligned in parallel [8]. In this case, flexible isolation layers will be required to prevent the interference between PVDF layers. The 
maximum number of layers depends on the thickness and rigidity of PVDF film in order to be rolled and fitted into biopsy needle.

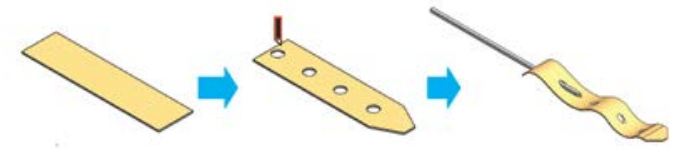

(a)

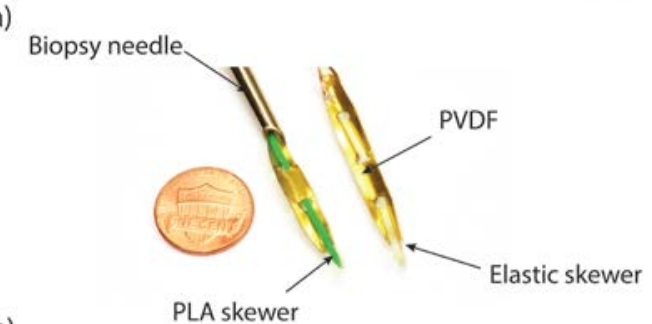

(b)

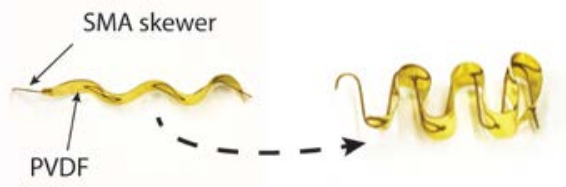

(c)

Figure 2: (a) Fabrication process; (b) fabricated prototype with PLA and elastic skewer in a biopsy needle; (c) temperature-shaped $P V D F$ with SMA skewer.

\section{RESULTS AND DISCUSSION}

An in-vitro experiment setup was used to characterize the vibration behavior as well as the output level of PVDF strip, Figure 3(a). A function generator (33220A, Agilent, USA) was connected to a power amplifier (XTi 1000, Crown Audio, USA) to drive a speaker for the generation of a controllable acoustic wave. In order to increase the deflections, PVDF, an impactor pointing at the center of the PVDF strip was attached to the speaker. To mimic the soft tissue (acoustic impedance $Z=1.5$ MRayls), 1.0\% agarose gel was used to house the PVDF strip. A digital multi-meter was used to read the output voltage from the PVDF. Assuming a simple strip forming a cantilever beam, the resonance frequencies can be given by

$$
f_{n}=\frac{k_{i}}{2 \pi} \sqrt{\frac{E I}{\rho W t L^{4}}}
$$

where $L$ is the length of the beam, $\rho$ is the density of the material, $\mathrm{W}$ is the width of the beam, $\mathrm{t}$ is the thickness of the material, $I$ is momentum of inertia and $E$ is Young's modulus, and $k_{i}$ is 3.52 for the $1^{\text {st }}$ resonance. A frequency sweep was initially conducted to locate the resonant frequency, indicating $250 \mathrm{~Hz}$ for a $1 \times 2 \mathrm{~cm}^{2}$ PVDF strip.

Once the resonant frequency was determined, the electric output characterization was studied with different boundary conditions to simulate possible implantation situations. First, a planer PVDF in $1 \times 2 \mathrm{~cm}^{2}$ dimension was submerged in a water balloon with the vibration delivered to the water. Thus, water is the acoustic coupling media for conducting the acoustic vibration and can also dampen the motion of PVDF, Figure 3(b); such configuration has been described for an acoustically powered transponder [9]. To compare with the first case, by using the same setup as shown in Figure 3(a), the PVDF of the same dimension was also tested in a piece of $1.0 \%$ agarose gel (Sigma-Aldrich, USA) with the thickness of $5 \mathrm{~mm}$ between the impactor and the PVDF.

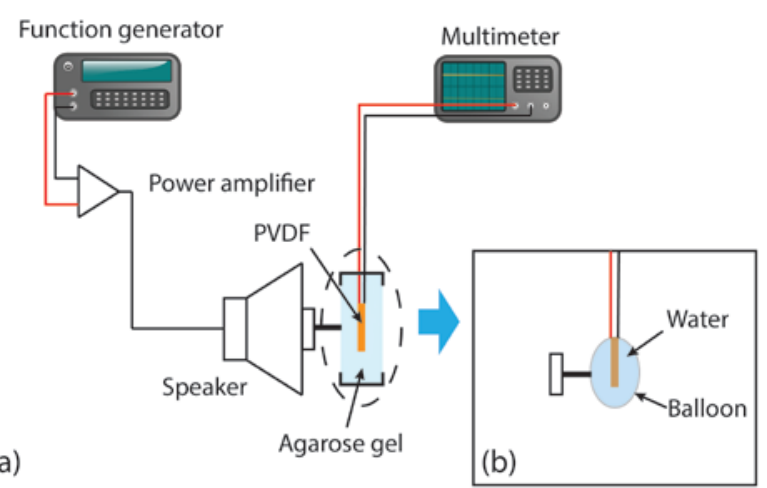

Figure 3: Schematic of in-vitro experiment setup: a function generator is connected to a power amplifier for driving the speaker and impactor. Device is placed in different situations for measuring the output: (a) inside agarose gel slab; (b) bending vibration in water.

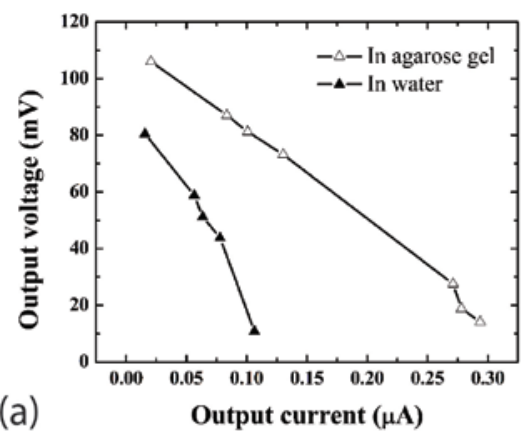

(b)

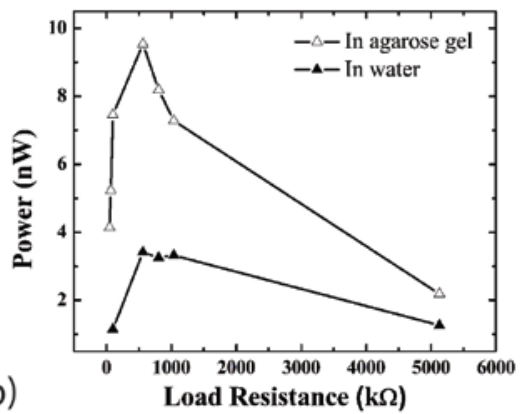

Figure 4: Electric output characterization under different conditions: (a) Output I-V curve with different load resistances; (b) Output power curve with different load resistances.

Figure 4(a) depicts the output I-V curve from single layer PVDF $\left(1 \times 2 \mathrm{~cm}^{2}\right)$ when different load resistors were applied. For the output characterization, a $92 \mathrm{~dB}$ acoustic intensity at $250 \mathrm{~Hz}$ was applied at the surface of the speaker to provide sufficient acoustic pressure for powering. As shown in the plot, PVDF vibrating in the agarose gel produces a higher displacement and more charges as well. A higher output power at different loads are generated than the case in water. Water has been commonly used as coupling media for most ultrasonic therapeutic applications due to its ideal acoustic characteristics [10]; however, in our experiment setup, the reflection at the water/air interface (reflection would be $99.9 \%$ [11]) causes the 
wave pattern inside the spherical container to become less uniform along the PVDF's poling direction (compared with the gel), which results in a lower output power. Figure 4(b) illustrates the output power with different load resistances. The maximum output power can be detected with a load resistance of $562 \mathrm{k} \Omega$ for the case when the PVDF is in the agarose gel and the output power is $\sim 9.5 \mathrm{nW}$. The output power decreases dramatically when the load resistance is smaller than $100 \mathrm{k} \Omega$. The output power reduces by $56 \%$ when the load is $48 \mathrm{k} \Omega$.

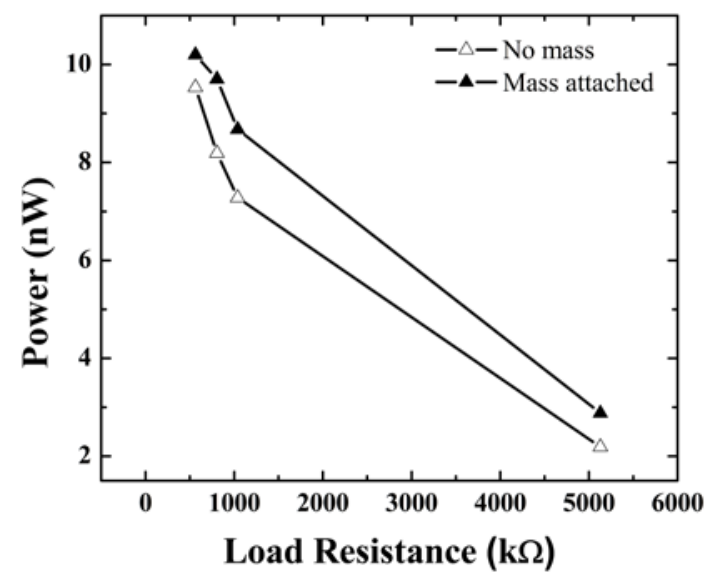

Figure 5: Electric output characterization under different conditions: (a) Output I-V curve with different load resistances; (b) Output power curve with different load resistances.

In order to increase the displacement while implanted, a mass of $0.11 \mathrm{~g}$ was attached to the same PVDF strip. The resonant frequency was shifted to $135 \mathrm{~Hz}$ due to the increase of the total weight of the cantilever and the difference in driving capability is compared in Figure 5. For the measurement, the same acoustic input (92dB) was chosen at different resonant frequency. The peak output power was measured to be $10.2 \mathrm{nW}$ which is $7.4 \%$ higher when mass is added. The output curve of the original PVDF is only shifted upwards. Thus, the application of a small mass can increase the output power without changing the driving behavior of PVDF. A higher output and a lower resonant frequency is expected when a heavier mass is attached. It is important to note that for the simplicity of implantation, the width of the mass cannot exceed the diameter of the biopsy needle $(2 \mathrm{~mm})$ and a mass in smaller size can also introduce less boundary constraint on the PVDF.

Based on the characterization, local delivery of acoustic vibration and an additional mass are preferred for the optimization of output power. The output of different PVDF piezoelectric power sources are summarized in Table 1 . Without sacrificing the feasibility of easy implantation, the output can also be increased further if PVDF is larger in size and stacked to form a multi-layer structure. Output impedance matching circuitry can also be integrated with powered electronic system to amplify the output power delivered to the load.

As a proof-of-concept, the capability of using the design for energy harvesting from low-frequency human motion/impact was also validated. The output from a series of soft-impacts on PVDF was measured inside water with an interval of 1 second. The softimpact was applied periodically by a single finger tapping motion. As shown in Figure 6, once the PVDF was bended, the releasing of finger and an inverse displacement create two voltage peaks in different polarizations. The vibration of PVDF in water lasts for around 0.1 second. The maximum output power at the peak is calculated to be $1.58 \mathrm{nW}$ which is $25 \%$ higher when the speaker is used to generate a continuous output. The tissue motion during the normal activity can provide a higher displacement and the electric energy generated can be stored using a rectifier and capacitors.

Table 1: Output power in different PVDF energy sources [8][12$13]$.

\begin{tabular}{cccc}
\hline \hline Device & $\begin{array}{c}\text { Dimension } \\
(\mathrm{mm})\end{array}$ & Input level & $\begin{array}{c}\text { Output } \\
(\mathrm{nW})\end{array}$ \\
\hline $\begin{array}{c}\text { PVDF cantilever } \\
\text { in Helmholtz } \\
\text { resonator }\end{array}$ & $15 \times 10.6 \times 0.37$ & $\begin{array}{c}118 \mathrm{~dB} \\
(15 \mathrm{~Pa})\end{array}$ & 190 \\
$\begin{array}{c}\text { PVDF beam in } \\
\text { sonic crystal } \\
\text { PVDF bimorph } \\
\text { cantilever }\end{array}$ & $50 \times 12 \times 0.028$ & $7 \mathrm{~Pa}$ & $35-40$ \\
$\quad$ This work & $20 \times 10 \times 0.11$ & $92 \mathrm{~dB}$ & $1.5 \mathrm{~g}$ \\
\hline \hline
\end{tabular}

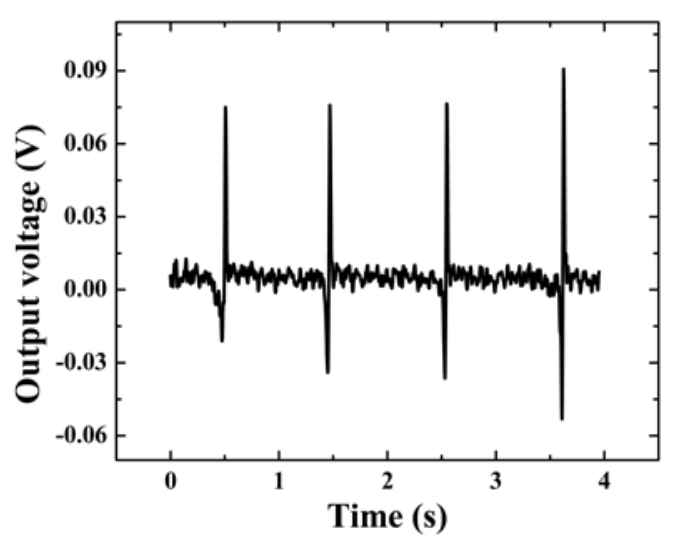

Figure 6. Output voltage when the PVDF is receiving a series of soft-impacts in water.

\section{CONCLUSION}

We presented an acoustically-powered implantable PVDF power source for low power implantable devices. The designed configuration as well as fabrication process make it possible for lowcost manufacturing and reshaping after implantation. The device was characterized in vitro using a variety of acoustic and impact forces in tissue simulating environments. The designed form factor allows the PVDF to fit in a biopsy needle. A shape memory alloy wires used in a skewer configuration provides in situ shaping once the device is inside the body at $37^{\circ} \mathrm{C}$.

\section{ACKNOWLEDGEMENTS}

The authors thank the staff at Purdue University Birck Nanotechnology Center and the Ziaie Biomedical Microdevices Laboratory members for their assistance in fabrication and experiments. This work is partially supported by the National Institutes of Health.

\section{REFERENCES}

[1] P. D. Mitcheson, E. M. Yeatman, G. K. Rao, A. S. Holmes, and T. C. Green. "Energy harvesting from human and machine 
motion for wireless electronic devices." Proceedings of the IEEE 96, no. 9 (2008): 1457-1486.

[2] Takeuchi, Esther S., and Randolph A. Leising. "Lithium batteries for biomedical applications." MRS bulletin 27.08 (2002): 624627.

[3] Schmidt, Craig L., and Paul M. Skarstad. "The future of lithium and lithium-ion batteries in implantable medical devices." Journal of power sources 97 (2001): 742-746.

[4] Catrysse, Michael, Bart Hermans, and Robert Puers. "An inductive power system with integrated bi-directional datatransmission." Sensors and Actuators A: Physical 115.2 (2004): 221-229.

[5] S. H. Song, A. Kim, S. Member, B. Ziaie, and S. Member, "Omni - Directional Ultrasonic Powering for Millimeter - Scale Implantable Devices," pp. 1-8, 2015.

[6] J. Zhou, A. Kim, S. H. Song, and B. Ziaie, "An ultrasonically powered implantable micro-light source for localized photodynamic therapy." Solid-State Sensors, Actuators and Microsystems (TRANSDUCERS), 2015 Transducers-2015 18th International Conference on. IEEE, 2015.

[7] A. Denisov and E. Yeatman, "Ultrasonic vs. Inductive Power Delivery for Miniature Biomedical Implants," 2010 Int. Conf. Body Sens. Networks, pp. 84-89, Jun. 2010.
[8] H. Y. Lee and B. Choi, "A multilayer PVDF composite cantilever in the Helmholtz resonator for energy harvesting from sound pressure," Smart Mater. Struct., vol. 22, no. 11, p. 115025, 2013.

[9] A. Kim, C. R. Powell, and B. Ziaie, "An implantable pressure sensing system with electromechanical interrogation scheme," IEEE Trans. Biomed. Eng., vol. 61, no. 7, pp. 2209-2217, 2014.

[10] A. F. Prokop, S. Vaezy, M. L. Noble, P. J. Kaczkowski, R. W. Martin, and L. A. Crum, "Polyacrylamide gel as an acoustic coupling medium for focused ultrasound therapy," Ultrasound Med. Biol., vol. 29, no. 9, pp. 1351-1358, 2003.

[11] J. E. Aldrich, "Basic physics of ultrasound imaging," Critical Care Medicine, vol. 35, no. 5 Suppl, pp. S131-7, May 2007.

[12] W.-C. Wang, L.-Y. Wu, L.-W. Chen, and C.-M. Liu, "Acoustic energy harvesting by piezoelectric curved beams in the cavity of a sonic crystal," Smart Mater. Struct., vol. 19, no. 4, p. 045016, 2010.

[13] J. Eom, J. Lee, H. Lee, and B. Choi, "Energy harvesting for bladder pressure sensor using parametric amplification phenomenon of PVDF bimorph cantilever," BMEiCON 2013 6th Biomed. Eng. Int. Conf., pp. 13-15, 2013.

\section{CONTACT}

* B. Ziaie; bziaie@purdue.edu 